\title{
Room temperature water Leidenfrost droplets
}

\author{
Franck Celestini ${ }^{1}$, Thomas Frisch ${ }^{2}$, Yves Pomeau ${ }^{3}$ \\ 1 Laboratoire de Physique de la Matière Condensée, CNRS UMR 7366, \\ Université de Nice Sophia-Antipolis, Parc Valrose 06108 Nice Cedex 2, France \\ 2 Institut Non Linéaire de Nice, CNRS UMR 7735, Université de Nice Sophia-Antipolis, \\ 1361 Routes des lucioles, Sophia Antipolis F-06560 Valbonne France and \\ 3 University of Arizona, Department of Mathematics, Tucson, AZ 85721 USA
}

\begin{abstract}
We experimentally investigate the Leidenfrost effect at pressures ranging from 1 to 0.05 atmospheric pressure. As a direct consequence of the Clausius-Clapeyron phase diagram of water, the droplet temperature can be at ambient temperature in a non-sophisticated lab environment. Furthermore, the lifetime of the Leidenfrost droplet is significantly increased in this low pressure environment. The temperature and pressure dependance of the evaporation rate are successfully tested against a recently proposed model. These results may pave a way to reach efficient Leidenfrost micro-fluidic and milli-fluidic applications.
\end{abstract}

PACS numbers: 47.55.D-, 68.03.-g

There has been lately numerous studies of the dynamical properties of Leidenfrost droplets [1 $[$, , 8]. Undoubtedly one of the reasons of this interest is in the possible applications in micro-fluidic and milli-fluidic. These droplets float on a thin vapor film which strongly delays their evaporation rate due to the low thermal heat conductivity of the vapor as compared to the one of the liquid. The transport by Leidenfrost droplets has the big advantage, compared to regular microfluidic in small pipes, to be without contact with solid surfaces which are oftenly a potential source of pollution. However this advantage of no contact with the surfaces is plagued by other inconveniences. Among them, the temperature inside water Leidenfrost droplets is usually too high and is in the vicinity of the boiling temperature of the liquid they are made of. In particular, the temperature water near boiling at atmospheric room pressure is often far above the range of stability of most biomolecules which could be contained in the droplet. The investigation we report below is devoted to a simple idea, namely the one of using a low pressure environment to lower the boiling temperature to the room temperature. This may pave the way to potential micro-fluidics and milli-fluidic devices using Leidenfrost droplets as small room temperature reactors. Since the boiling temperature of water falls rather sharply as pressure decreases, it is in principle not difficult to reach pressures where most biomolecules would survive in Leidenfrost droplets. However, since the Leidenfrost effect on droplets is a rather complex phenomenon [7], we found it useful to check that it still exists at low pressure and low temperatures with water droplets. Moreover, the controlled formation and smooth release of small spherical droplets in low pressure situations is not such a trivial task, at least without recourse to complex laboratory methods.

We present in this Letter a realization using fairly standard methods which are likely easily reproducible in a non too sophisticated lab environment. We first put forth our experimental set-up and the results obtained for the evolution of the droplet radius as function of time in low pressure conditions. We experimentally examine the mass evaporation rate as a function of the applied pressure $P$ and of the temperature difference between the Leidenfrost droplet and the heated plate on which it is levitating. We put in evidence a significant increased lifetime of a low pressure Leidenfrost droplet. These results are successfully compared with the recently proposed theoretical analysis of the Leidenfrost phenomena [7]. We finally conclude this paper by pointing out the possible applications of this room temperature Leidenfrost effect.

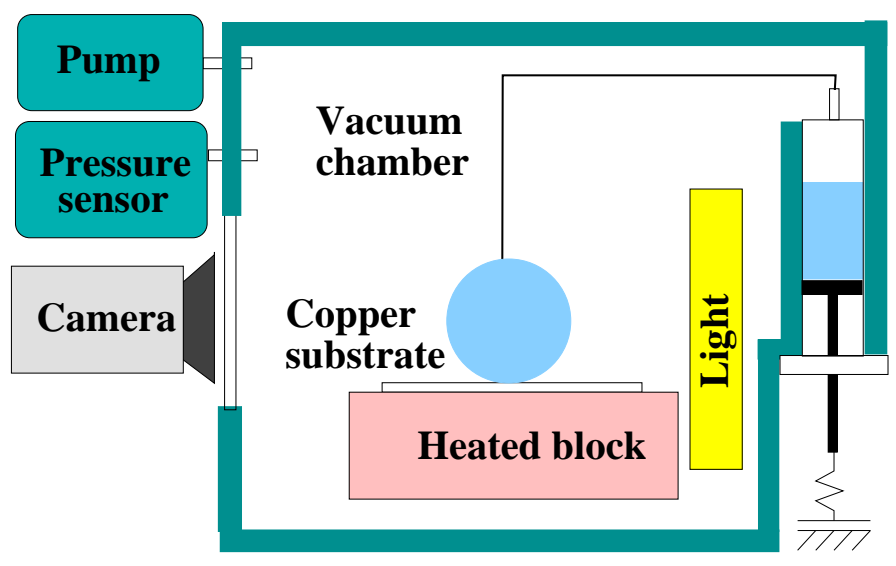

FIG. 1: Sketch of the experimental set-up.

The experimental set-up is displayed in Fig. 1 A primary vacuum pump is used to impose the pressure $P$ in the chamber of volume $10^{-2} \mathrm{~m}^{3}$. A differential pressure sensor measures accurately the pressure $P$ in the 1000 1 mbar range. The droplets are deposited on the substrate with a syringe inserted in the vacuum chamber in a vertical position. This last point is of importance since it permits to evacuate gas bubbles dissolved in the water which appear when the pressure is lowered. The temper- 
ature of the droplet is denoted $T$. As for the classical (atmospheric pressure) Leidenfrost effect, the liquid is at thermal equilibrium with the vapor. As a consequence the value of $T$ follows from the pressure measurement and the classical water liquid-vapor phase diagram $(\mathrm{P}-$ $\mathrm{T})$. The substrate is a polished copper block heated at a controlled temperature $T_{s}$ and the temperature difference between the heated plate and the droplet is denoted $\Delta T=T_{s}-T$. The substrate temperature is directly measured using a temperature sensor inserted in the heated block. A glass window in the vacuum chamber permits the observation of the droplet with a camera. Once the droplet is deposited on the substrate, we record the decrease of its radius $R(t)$ due to the evaporation on the heated substrate. We deduce the volume of the droplet and the mass evaporation rate $J \propto \rho_{l} \frac{\partial R^{3}}{\partial t}$. Here we will focus on the results obtained for droplet with radius lower than the capillary length $R_{c}=\left(\frac{\sigma}{\rho_{l} g}\right)^{1 / 2}, \sigma$ being the surface tension between the liquid and the vapor, $\rho_{l}$ the mass density of the liquid and $g$ the acceleration of gravity.

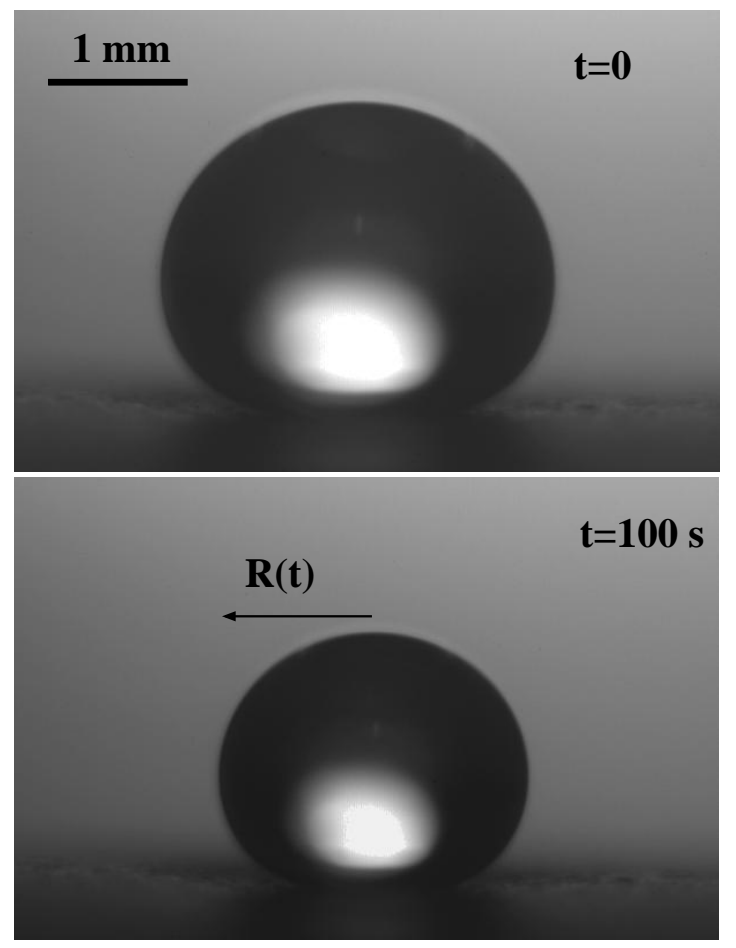

FIG. 2: View from the side of the Leidenfrost droplet for $t=$ $0 s$ and $t=100 s$ at a pressure of $1 / 20$ atmosphere $(50 \mathrm{mbar}$ ) . At this pressure, the temperature of the Leidenfrost droplet is 33 Celsius.

As shown on Fig. 2. we observe a Leidenfrost droplet under a pressure of 50 mbar (1/20 atmosphere). It has the shape of a weakly deformed sphere as expected for a droplet of radius smaller than the capillary length. This droplet floats on a thin vapor film which has a small thermal conductivity compared to the one of the liquid. The droplet is at a temperature $T$ corresponding to the

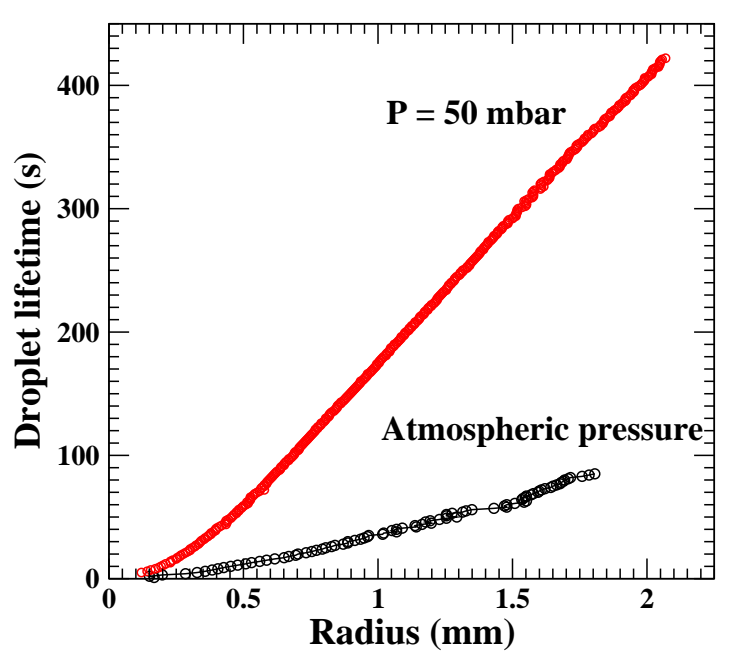

FIG. 3: Leidenfrost droplet life-time as function of the radius for two different pressure: top red (color on-line) $P=50$ mbar, bottom black $P=1$ bar. The temperature of the substrate is 120 Celsius for the top red curve and 250 Celsius for the bottom black curve.

vapor-liquid equilibrium temperature at a given pressure. For the droplet represented in Fig. 2, the pressure $P$ is set to 50 mbar thus leading to a droplet temperature of 33 Celsius. As stressed above the main characteristic of a Leidenfrost droplet under a controlled pressure environment is that for sufficiently low pressure the drop can be at room temperature. Furthermore, another attribute of the droplet is the noticeable increase of its lifetime. For a given initial radius $R$, this lifetime is simply defined as the time for the droplet to reach a radius that is no longer observable with our optical device. This increase is illustrated in Fig. 3 for two different values of the pressure. Indeed, the mass evaporation rate is related to the temperature difference between the droplet and the hot plate as expressed in equation (1) below. The temperature difference $\Delta T$ between the substrate and the droplet is of 87 Celsius for the 50 mbar case and is of of 150 Celsius for the 1 bar case. This smaller temperature gap at low pressure is a factor which contributes to the increases the life time of the droplet. As shown on Fig. 3, one can see that the lifetime of a low pressure Leidenfrost droplet is increased by a factor of order 10. For example, a droplet of initial radius equal to the capillary length $R_{c}$ can reach a lifetime of ten minutes.

This lifetime increase is not solely due to the pressure reduction but also to the temperature difference $\Delta T$ between the plate and the drop. Let us recall the recently published results for the mass evaporation rate $J=d M / d t$ of Leidenfrost droplets [7]. For droplets of radii ranging between an "intermediary length" $R_{i}=$ $\left(R_{l}^{3} R_{c}^{4}\right)^{1 / 7}[13]$ and the capillary length $R_{c}$ it has been shown that $J$ scales as :

$$
J \propto R^{12 / 5} P^{1 / 5} \Delta T^{4 / 5} .
$$


This equation relates the mass evaporation rate to the radius of the droplet, to the applied pressure and to the temperature difference between the droplet and the hot plate. Equation (1) has been derived from a theoretical analysis using the lubrication limit to model the vapor flow below the Leidenfrost droplet [7]. It is worth noticing that this prediction is more elaborated than the standard approach of the Leidenfrost phenomenon [9] that predicts $J \propto R \Delta T$ without any dependance on the applied pressure. In Eq.(1), the $P^{1 / 5}$ dependance arises from the linear dependance of the vapor density with the pressure at constant temperature [14].

To verify this prediction we first perform experiments to ascertain our scaling with respect to $\Delta T$. The pressure in the vacuum chamber is set to 75 mbar and three evaporation rates are recorded for three different value of the temperature difference: $\Delta T=80,120,150$ Celsius. As shown on Fig. 4, we plot $J / \Delta T^{4 / 5}$ as a function of the droplet radius. One can see that the data sets collapse on a same curve. Thus this strongly validates the $\Delta T^{4 / 5}$ dependance of $J$ given by Eq. (11). The best fit of our experimental data leads to $J / \Delta T^{4 / 5} \propto R^{2}$ which also lies in close agreement with our prediction of $J / \Delta T^{4 / 5} \propto R^{2.4}$. The relative agreement between the theory and the experiments is not surprising since the theory is made upon the lubrication approximation. A more accurate theoretical analysis would required the full resolution of Navier-Stokes for a biphasic system (liquidvapor). It is important to note that this results hold for $R$ lying between $R_{i}$ and $R_{c}$ and that another scaling law may appear in different regimes $\left(R>R_{c}\right.$ or $\left.R_{l}<R<R_{i}\right)$.

We now look at the dependance of the mass evaporation rate $J$ with respect to the pressure. We set the value of $\Delta T$ to $80 \mathrm{~K}$ and perform three experiments for different values of the pressure : $P=50 \mathrm{mbar}, 250 \mathrm{mbar}$ and 500 mbar. As shown on Fig. 5, we plot the pressure normalized mass evaporation rate $J / P^{1 / 5}$ as a function of $R$. As for the previous analysis, these data collapse on the same curve, scaling as $R^{2}$, evidencing a good agreement between the experiments and theory.

We have demonstrated in this Letter that Leidenfrost droplets can be generated at room temperature using a non too sophisticated lab environment. The life-time of the droplets increases by roughly one order of magnitude compared to the standard atmospheric pressure conditions. The evaporation rates is decreased at low pressure due to the small difference of temperature between the substrate and the droplet. We have also verified that the scaling law for the mass evaporation rate as a function of the temperature, pressure and radii agree with our recently proposed theory. We hope that this study will stimulate discussion and interest as a possible device in the micro and milli-fluidic area in which hovering water droplet at room temperature may serve as bio- or chemio-reactors $10-12$.

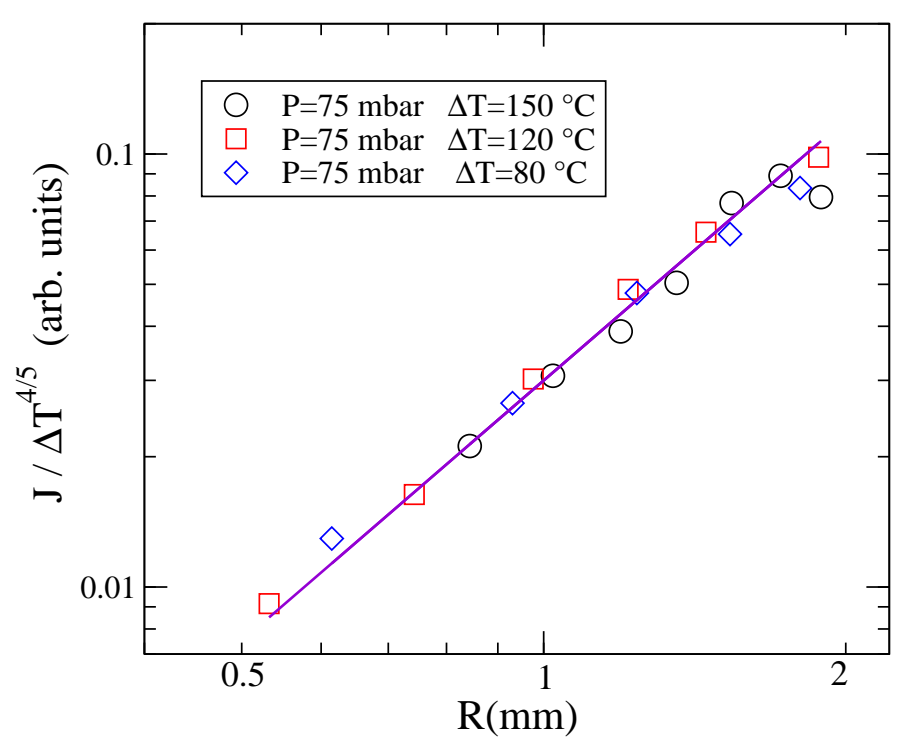

FIG. 4: Temperature normalized mass evaporation rate versus radius at constant pressure $p=75 \mathrm{mbar}$ for three different value of $\Delta T=80,120,150$ Celsius. The best fit (full blue line, color on-line) leads to $R^{2}$ tendency which is close to the the-

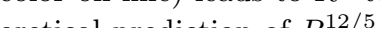

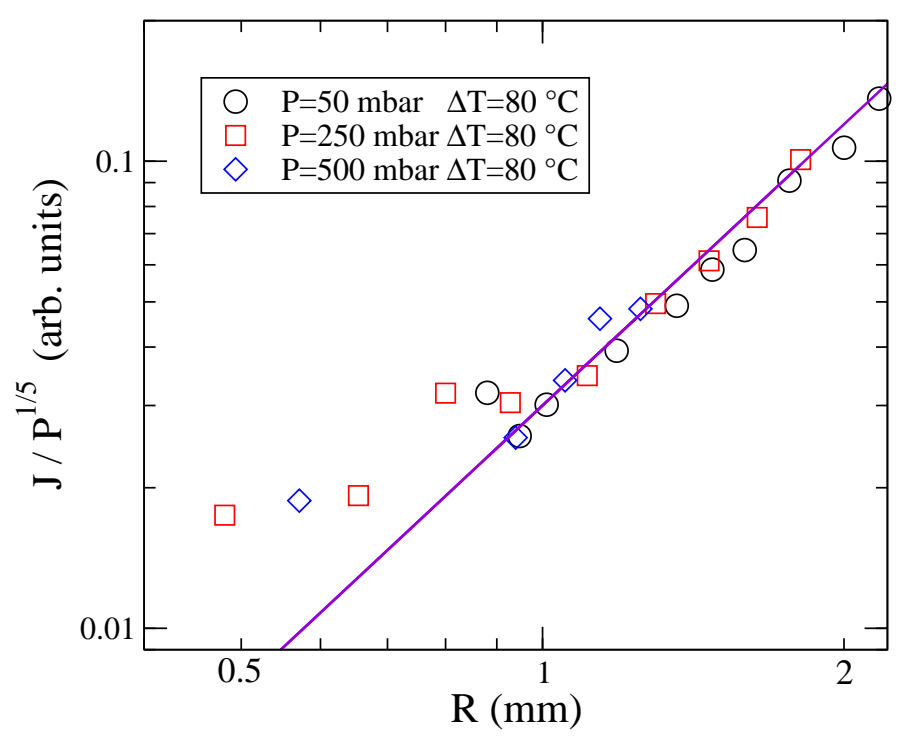

FIG. 5: Pressure normalized mass evaporation rate versus radius at constant temperature $\Delta T=80$ Celsius for three different values of the pressure : $P=50 \mathrm{mbar}, 250 \mathrm{mbar}$ and 500 mbar. The best fit (full blue line, color on-line) leads to $R^{2}$ tendency which is close the theoretical prediction of $R^{12 / 5}$ as in the previous figure.

We would like to thank Jean-Pierre Romagnan and Patrick Tabeling for fruitful discussions. 
[1] F. Celestini, T. Frisch and Y. Pomeau, Phys. Rev. Lett. 109, 034501 (2012)

[2] J.C. Burton, A.L. Sharpe, R.C.A. van der Veen, A. Franco and S. R. Nagel, Phys. Rev. lett. 109, 074301 (2012).

[3] F. Celestini and G. Kirstetter, Soft Matter 8, 5992 (2012)

[4] J H. Linke, B. J. Aleman, L. D. Melling, M. J. Taormina, M. J. Francis, C. C. Dow-Hygelund, V. Narayanan, R. P. Taylor1, and A. Stout, Phys. Rev. lett. 96, 154502 (2006).

[5] D. Quéré, Ann. Rev. Fluid. Mech. 45, 197 (2013).

[6] T. R. Cousins, R. E. Goldstein, J. W. Jaworski and A. I. Pesci. J. Fluid. Mech. 696, 215 (2012).

[7] Y. Pomeau, M. Le Berre, F. Celestini and T. Frisch, C. R. Mecanique 340, 867 (2012).

[8] X. Xu and T. Qian, Phys. Rev. E 87, 043013 (2013).

[9] A-L. Biance, C. Clanet and D. Quéré, Phys. Fluids., 15, $1632(2003)$

[10] B. J. Kirby, Micro- and Nanoscale Fluid Mechanics: Transport in Microfluidic Devices, Cambridge University Press, (2010)

[11] P. Tabeling. Introduction to Microfluidics. Oxford Uni- versity Press. (2005)

[12] L. Baraban, F. Bertholle, M. L.M. Salverda, N. Bremond, P. Panizza, J. Baudry, J. A. G.M. de Visser and J. Bibette, Millifluidic droplet analyser for microbiology. Lab Chip, 11, 4057, (2011)

[13] The radius $R_{l}=\left(\frac{\eta \Delta T \lambda}{g L \rho_{v} \rho_{l}}\right)^{1 / 3}$ represent the lubrication limit below which the lubrication approximation break down and the droplet levitates and rises [1, 7, 8]. Here $\lambda$ is the thermal conductivity, $L$ is the latent heat, $\eta$ is he air dynamic viscosity and $\sigma$ the water surface tension. The radius $R_{i}=\left(R_{l}^{3} R_{c}^{4}\right)^{1 / 7}=100$ microns is another characteristic length he below which the pressure induced by the Poiseuille flow below the drop is lower than the Laplace pressure. As a consequence for $R<R_{i}$ the droplet turns in a quasi-spherical shape.

[14] The derivation of this results uses the formula $J \propto$ $\frac{\lambda \Delta T}{L}\left(\frac{R^{12 / 5}}{R_{c}^{45} R_{l}^{3}}\right)^{1 / 5}$ which can be found in equation (44) of reference [7]. The thermal conductivity of the vapor and its viscosity remains quasi-independent of the pressure as expected from the law of perfect gas. However the $\rho_{v} \propto P / K_{b} T$ dependency in the value of $R_{l}$ given in the previous footnote leads to a scaling $P^{1 / 5}$ in equation (1) for the evaporation flux. 